\title{
Stimulating Cooperative Diversity in Wireless Ad Hoc Networks through Pricing
}

\author{
Naveen Shastry and Raviraj S. Adve \\ Department of Electrical and Computer Engineering, University of Toronto \\ 10 King's College Road, Toronto, Ontario, M5S 3G4, Canada \\ Email: $\{$ naveen. shastry@utoronto.ca, rsadve@comm.utoronto.ca\}
}

\begin{abstract}
This paper addresses the issue of stimulating cooperative diversity among selfish nodes in commercial wireless ad hoc networks. For the relay, cooperation represents both a real cost of energy expenditure and an opportunity cost of possible delays for its own data. Since nodes are selfish, we propose a pricing game that stimulates cooperation via reimbursements to the relay. Specifically, given the price per channel use, the source and relay interact through reimbursement prices, transmitter power control and forwarding/protocol preferences such that their utilities are maximized. Our pricing game is shown to converge to a Nash equilibrium where cooperative diversity is induced at intuitively reasonable network geometries.
\end{abstract}

\section{INTRODUCTION}

A wireless ad hoc network consists of a set of nodes that are organized and maintained in a distributed manner. Applications of such networks include battlefield communications, disaster relief, extension of access point (AP) provision, etc. In such networks, node cooperation significantly increases system performance. "Cooperation" refers to a node's willingness to sacrifice resources (e.g., energy, bandwidth) for the benefit of other nodes in the network. In applications of ad hoc networks for military and disaster relief, cooperation among nodes can be assumed since the nodes belong to a single authority and thus voluntarily cooperate to achieve a common goal. However, in commercial applications there is no good reason to assume that nodes will cooperate. In fact, given that nodes are independent entities, nodes are selfish, i.e., nodes consume their resources solely to maximize their own benefit.

This paper focuses on stimulating nodes to participate in cooperative diversity transmissions in commercial wireless ad hoc networks. Cooperative diversity has been been shown to significantly enhance system performance in comparison to both direct and multi-hop transmissions [1], [2]. Typically in ad hoc networks, nodes are battery powered and bandwidth constrained and cooperation implies both a real, in power expended, and opportunity cost, in lost transmissions. A selfish node therefore needs an incentive to cooperate [3]. Researchers have developed several approaches to stimulate packet forwarding (multi hop transmission) in these networks. These approaches can be classified as either reputation-based [4][6] or pricing-based systems [7]-[11]. We focus on the latter approach. To our knowledge, this is the first paper to analyze pricing in the context of cooperative diversity.

In pricing-based systems, a node receives payment (reimbursements) for forwarding packets for others which can then be used for transmitting one's own data in the future. Buttayan and Hubaux [7] and Zhong et al. [8] develop pricingbased protocols where the amount charged per packet is determined exogenously and is the same for each node in the network. Crowcroft et al. [9] propose a scheme where the nodes determine the price per packet forwarded allowing them to dynamically update their price for resources based on their bandwidth and battery level. Marbach and Qiu [10] provide a formal analysis for the above setting, including the existence of an equilibrium, the degree of cooperation attained under equilibrium and successful convergence to equilibrium strategies. The drawback with the above approaches is the assumption of a simplified channel model - the energy required to forward a packet is assumed to be constant regardless of transmission distance.

Ileri et al. were the first to develop a stimulation mechanism that takes into account the fading channel [11]. The authors study the interaction between multiple nodes and a revenue seeking access point (AP). The AP and nodes use a joint maximization approach. Their analytical and simulation results show that cooperation is highly dependent on network geometry. Their cooperation scheme works best in two-hop chains where both nodes are relatively close to each other.

One important drawback with the work in [11] is that, in some network scenarios, a relay node's utility with forwarding is lower than that achieved in a non-forwarding system. This is because the stimulation mechanism is dominated by the revenue maximizing AP. Thus in certain network settings, the pricing mechanism benefits only the AP, not the nodes.

This paper extends the work in [11] to packet forwarding with cooperative diversity. The goal in our mechanism is to not only induce forwarding at reasonable network geometries, but to ensure that both the AP and users (the terms "node" and "user" are used interchangeably) benefit from cooperation. Specifically, we design a pricing scheme in which the AP charges users for transmitting data packets and users reimburse each other for forwarding. Initially, the AP declares a set of service prices, one for each source-relay pair in the network. These prices are chosen such that the AP's revenue is maximized for the non-forwarding network. Note that the concept of "service" is related to the amount of data transmitted by the user. The effective network therefore reduces to two nodes (one source, one potential relay) and an AP. Figure 1 illustrates the pricing scheme. Given the AP's service price and the 


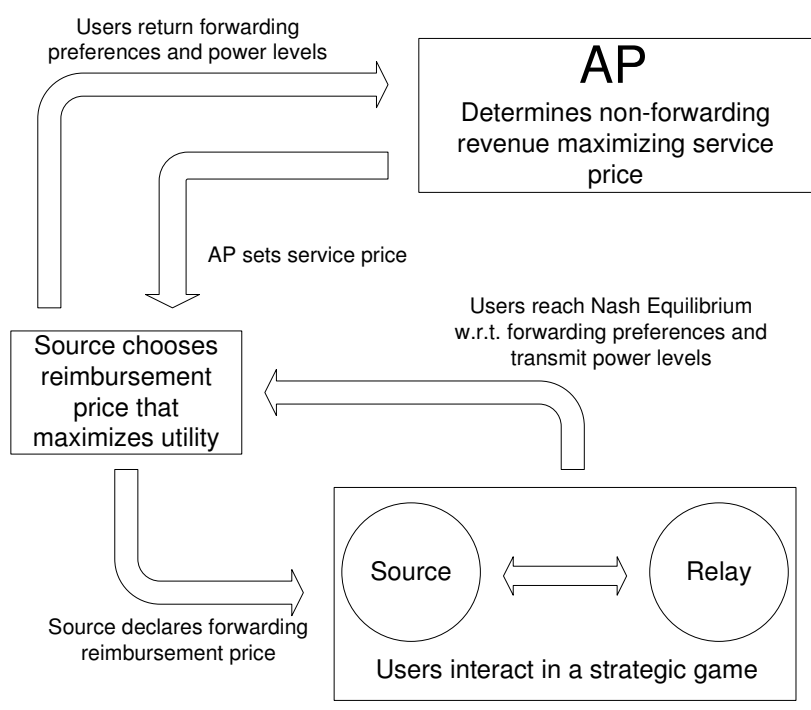

Fig. 1. Illustration of Pricing Scheme for a 2 user one AP network

source's reimbursement price for forwarding, the interaction between users is a strategic game, i.e., a simultaneous move game where players chose actions without knowing the current actions of the other players [12]. Specifically in our case, the users are responsible for choosing their transmit power level and deciding on whether to cooperate. The game between the users reaches a Nash equilibrium when no user can unilaterally deviate from its strategy and still increase its utility. The source node executes an optimization algorithm to maximize its utility. The pricing mechanism converges at the reimbursement price that maximizes the source node's utility. The scheme proposed here is simple, distributed and scalable.

This paper is organized as follows. Section II presents the system model used and defines the utility functions used. Section III analyzes the game set up in Section II. Section IV presents simulations illustrating the performance of the proposed scheme. The paper ends with conclusions in Section V.

\section{SySTEM MODEL}

The network here comprises a fixed set of users (nodes) communicating with a single AP, the final destination for all user transmissions. Each user is assumed to have data of its own to transmit, organized in a packet of $M$ bits. Each user's transmission is assumed to be interference free. The network is assumed static in the sense that the convergence time of the pricing mechanism is shorter than both the coherence time of the channel and variations in user mobility.

The functionality of the proposed scheme is dependent on the initial routing assignments in the network. We assume very simple routes, where users are clustered in groups of two according to their proximity. For each cluster, the node closer to the AP is designated as the "potential relay". The other user in the group is labelled as the "source". Despite these rather simplistic assumptions regarding routing, the model provides significant insight towards the implementation of cooperative diversity transmission in pricing based systems.

\section{A. Communication Satisfaction Metric}

The first step in developing the game theoretic framework is to quantify a user's satisfaction in transmitting its own data independent of the pricing mechanism. Any function that correctly characterizes a user's preferences is an appropriate measure. In an ad hoc network, a user's communication merit depends on two factors: throughput and battery life. A selfish user would want to achieve the greatest throughput possible while expending the least amount of energy. Clearly, a trade off exists between achieving high throughput and low energy consumption. Saraydar et al. propose a utility function that quantifies the above trade off:

$$
U(p)=\frac{T(p)}{p} \text { bits/joule, }
$$

where $T(p)$ and $p$ are the user's throughput and transmit power respectively. The throughput is related to the power via the signal-to-noise ratio $(\gamma)$ which determines an efficiency function, borrowed from [13], defined below:

$$
\begin{aligned}
& T(p)=W * f(\gamma), \\
& f(\gamma)=[1-2 \operatorname{BER}(\gamma)]^{M}, \quad \gamma=\frac{h p}{N_{o} W},
\end{aligned}
$$

where $h, N_{o}$ and $W$ are the user's channel path gain, noise density and bandwidth respectively. The efficiency function $f(\gamma)$ approximates the true frame success rate (FSR)

$[1-\operatorname{BER}(\gamma)]^{M}$. Using this function allows for an accurate reflection of the user's preferences. In particular, as $p \rightarrow \infty$, $U(p) \rightarrow 0$ and as $p \rightarrow 0, U(p) \rightarrow 0$.

\section{B. User and AP Utility Functions}

In the proposed pricing mechanism, a user's utility is based on the role (relay or source) it plays in the network. The utility functions used here account for the satisfaction received in transmitting data and the associated AP charges. Depending on the user's role, the functions also include the real and opportunity costs with forwarding data along with the respective pricing rewards.

Before developing the utility functions, we first describe the action sets for each user in the game. For the potential relay, its action set is given by $\left\{p_{r}, k\right\}$, where $p_{r}$ is the transmit power level (bound by a maximum available power $p^{\max }$ ) and $k$ is the forwarding preference. Therefore, $p_{r} \in\left[0, p^{\max }\right]$ and $k$ is a binary indicator with $k=0$ the non-forwarding case and $k=1$ the case where the relay wants to cooperate with the source. The source's action set is specified by $\left\{p_{s}, l, \mu\right\}$, where $p_{s} \in\left[0, p^{\max }\right]$ is its transmit power level, $l$ is a binary indicator describing its choice in transmission protocol and $\mu$ is the reimbursement price that the source is willing to pay the relay for forwarding its data. Finally, a parameter $\lambda$ represents the AP's service charge per unit of data in the network.

Denote the path gains from the source to the AP, the source to the relay, and the relay to the $\mathrm{AP}$ as $h_{s a}, h_{s r}$ and $h_{r a}$ respectively. The channel throughputs from the source to the $\mathrm{AP}$, the source to the relay, and the relay to the AP are $T_{s a}=W f\left(\gamma_{s a}\right), T_{s r}=W f\left(\gamma_{s r}\right)$ and $T_{r a}=W f\left(\gamma_{r a}\right)$ with 
the index indicating the SNR of the corresponding channel. The throughput of the amplify-and-forward protocol is $T_{A F}=$ $W f\left(\gamma_{A F}\right)$, where $\gamma_{A F}$ is the received SNR that the source experiences over the AF channel. To simplify the analysis, we approximate this received SNR as

$$
\gamma_{A F}=\gamma_{s a}+\min \left\{\gamma_{s r}, \gamma_{r a}\right\}
$$

The user's utility is dependent on the type of forwarding protocol used in the network. This main theme of this paper is cooperative diversity using the amplify-and-forward protocol [2]. However, for completeness, we also develop the theory for the case where the relay forwards the source's data using multi-hop transmission. This is the only case that has been addressed earlier [11], though with some limitations. Section IV compares the two situations. This approach also helps understand the development of the utility functions used.

With multi-hop transmission as the forwarding protocol for the network, the source's end-to-end throughput (i.e., effective throughput) is limited by the amount of bandwidth that the relay devotes to forwarding. As a result the source's throughput is $\min \left\{T_{s r}, k T_{r a}\right\}$. The source's utility function is given by

$$
\begin{aligned}
& U_{s}^{m h}=W {\left[(1-l)\left(\frac{1}{p_{s}}-\lambda\right) f\left(\gamma_{s a}\right)-\lambda l f\left(\gamma_{s r}\right)\right.} \\
&\left.+\left(\frac{1}{p_{s}}-\mu\right) \min \left\{l f\left(\gamma_{s r}\right), k f\left(\gamma_{r a}\right)\right\}\right] .
\end{aligned}
$$

The first term in (5) corresponds to the non-forwarding case $(l=0)$ and the other terms to the case where the source transmits cooperatively $(l=1)$. The amount charged by the AP is proportional (via $\lambda$ ) to the amount of data transmitted by the source (either $T_{s a}$ for direct transmission or $T_{s r}$ for multi-hop transmission). Our pricing mechanism provides forwarding incentives through the reimbursement $\mu \min \left\{k T_{r a}, T_{s r}\right\}$.

The relay is reimbursed at a price proportional to the effective throughput received by the source. The relay's utility function is expressed as follows

$$
\begin{array}{r}
U_{r}^{m h}=W\left[(1-k)\left(\frac{1}{p_{r}}-\lambda\right) f\left(\gamma_{r a}\right)-\lambda k f\left(\gamma_{r a}\right)\right. \\
\left.+\mu \min \left\{k f\left(\gamma_{r a}\right), l f\left(\gamma_{s r}\right)\right\}\right] .
\end{array}
$$

Note, the relay pays service charges to the AP even when forwarding data for others. This is because the AP provides resources for radio links regardless of traffic characteristics.

Cooperative diversity is implemented here via the amplifyand-forward protocol. Since the AF protocol uses repetition coding, the source reimburses the relay by the amount $\mu T_{A F}$. Note that this reimbursement amount is proportional of the effective throughput experienced by the source. The source's utility function is given by:

$$
\begin{aligned}
U_{s}^{a f}=W[(1-l) & \left(\frac{1}{p_{s}}-\lambda\right) f\left(\gamma_{s a}\right) \\
& \left.+l\left(\frac{1}{p_{s}}-\lambda-\mu\right) f\left(\gamma_{A F}\right)\right] .
\end{aligned}
$$

If the relay prefers to forward data $(k=1)$, the AF protocol requires that the amount of data to be forwarded is $T_{A F}$. The relay's utility function is therefore expressed as

$$
U_{r}^{a f}=W\left[\left(\frac{1}{p_{r}}-\lambda\right)(1-k) f\left(\gamma_{r a}\right)+\mu k f\left(\gamma_{A F}\right)\right] .
$$

Within the pricing framework, the AP's level of satisfaction is determined by the revenue it generates from the network. We allow the AP to charge each user proportionally for the amount data transmitted over the network. We express the AP's revenue as the sum of the data transmitted by the source and relay of group $i$ multiplied by the price per unit of data $\lambda_{i}$. Mathematically, the utility function is given by

$$
U_{A P}=\sum_{i} \lambda_{i}\left(T_{s}^{i}+T_{r}^{i}\right) \text {. }
$$

\section{AnALYSIS OF PRICING MECHANISM}

We now analyze our scheme to show the existence of Nash equilibria and develop an iterative algorithm to converge to the most desirable equilibrium.

\section{A. User Optimizations}

Recall, that given the charge per unit of service, $\lambda$, and the reimbursement price for forwarding, $\mu$, the users engage in a strategic game to maximize their utilities unilaterally.

Given that the forwarding protocol is multi-hop transmission, the source and relay seek to maximize their utilities in (5) and (6) respectively. The $\min \{\}$ terms can be eliminated from both maximization problems by imposing the same additional constraints as in [11]. The optimization problem for the source and relay are as follows:

$$
\begin{array}{ll}
\max & U_{s}^{m h}\left(p_{s}, l\right) \\
\text { s. t. } & 0 \leq p_{s} \leq p^{\max } \quad l \in(0,1) \quad l f\left(\gamma_{s r}\right) \leq k f\left(\gamma_{r a}\right), \\
\max & U_{r}^{\text {mh }}\left(p_{r}, k\right) \\
\text { s. t. } & 0 \leq p_{r} \leq p^{\max } \quad k \in(0,1) \quad k f\left(\gamma_{r a}\right) \leq l f\left(\gamma_{s r}\right) .
\end{array}
$$

On the other hand, given that the forwarding uses the AF protocol, the source's optimization problem corresponds to the maximization of the utility function in (7),

$$
\begin{aligned}
\max & U_{s}^{\text {af }}\left(p_{s}, l\right) \\
\text { subject to } & 0 \leq p_{s} \leq p^{\max } \quad l \in(0,1), \quad l \leq k
\end{aligned}
$$

In the context of the AF protocol, the relay's optimization problem can be simplified by replacing the argument of the $\min \{\}$ term in (8) with $\gamma_{r a}$, with the added constraint that $\gamma_{r a} \leq \gamma_{s r}$. This is because if $\gamma_{r a}>\gamma_{s r}$, we can reduce $\gamma_{r a}$ (via reduction in $p_{r}$ ) and increase the relay's utility.

Using the utility function in (8), the optimization problem for the relay is

$$
\max \quad U_{r}^{a f}\left(p_{r}, k\right)
$$

subject to $\quad 0 \leq p_{r} \leq p^{\max }, k \epsilon(0,1), k \leq l, \gamma_{r a} \leq \gamma_{s r}$

To summarize, we model our strategic game as the simultaneous executions of user problems (12) and (13). This game is a function of the price per unit service, $\lambda$, and for cooperation, $\mu$. Both prices are exogenous. 


\section{B. Nash Equilibria of the Strategic Game}

Given $\lambda$ and $\mu$, if the individual nodes result in an action profile where each user's action is a best response to the other user's action, a Nash equilibrium is reached. In other words, a Nash equilibrium is the action profile $\left(p_{s}^{*}, l^{*}, p_{r}^{*}, k^{*}\right)$ where no user has an incentive to deviate by choosing another action given that the other user's action is fixed. The Nash equilibrium action profiles represent steady states in the game. Formally, the Nash equilibria are the following action profiles

For $\mathrm{MH}$ :

$$
\begin{aligned}
\left(p_{s}^{*}, l^{*}\right)= & \underset{ }{\arg \max } U_{s}^{m h}\left(p_{s}, l\right) \text { s.t. } 0 \leq p_{s} \leq p^{\max }, \\
& l(0,1), l f\left(\gamma_{s r}\right) \leq k^{*} f\left(\gamma_{r a}^{*}\right) \\
\left(p_{r}^{*}, k^{*}\right)= & \underset{k \in(0,1), k f\left(\gamma_{r a}\right) \leq l^{*} f\left(\gamma_{s r}^{*}\right)}{\arg \max U_{r}^{m h}\left(p_{r}, k\right) \text { s. t. } 0 \leq p_{r} \leq p^{\max },}
\end{aligned}
$$

For AF :

$$
\begin{aligned}
\left(p_{s}^{*}, l^{*}\right)= & \arg \max U_{s}^{a f}\left(p_{s}, l\right) \text { s.t. } 0 \leq p_{s} \leq p^{\max }, \\
& l \in(0,1), l \leq k^{*} \\
\left(p_{r}^{*}, k^{*}\right)= & \underset{\quad}{\arg \max U_{r}^{a f}\left(p_{r}, k\right) \quad \text { s. t. } 0 \leq p_{r} \leq p^{\max },}, \\
& k \in(0,1), k \leq l^{*}, \gamma_{r a} \leq \gamma_{s r}^{*}
\end{aligned}
$$

From (14), for multi-hop transmission, it is clear the equilibrium action profile must satisfy the opposing throughput constraints of the users' individual maximization problems. This opposing throughput constraint is $l^{*} f\left(\gamma_{s r}^{*}\right)=k^{*} f\left(\gamma_{r a}^{*}\right)$. Clearly, the non-forwarding action profile $\left(p_{s}^{*}, 0, p_{r}^{*}, 0\right)$ satisfies the above constraint and always exists in the game. We shall see in subsection IV that in certain network settings, a forwarding equilibrium also exists in our game. This equilibrium corresponds to the action profile $\left(p_{s}^{*}, 1, p_{r}^{*}, 1\right)$ that satisfies the throughput constraint $f\left(\gamma_{s r}^{*}\right)=f\left(\gamma_{r a}^{*}\right)$. We presents below an algorithm that converges to the forwarding equilibrium (if it exists).

For the amplify-and-forward protocol, the opposing constraints $l \leq k$ and $k \leq l$ imposed on the source and relay respectively result in Nash equilibria with the property $l=$ $k$. Again, the non-forwarding action profile $\left(p_{s}^{*}, 0, p_{r}^{*}, 0\right)$ is always a Nash equilibrium. As with the multi-hop setting, it is possible for a forwarding equilibrium to exist. This corresponds to the situation where the action profile is $\left(p_{s}^{*}, 1, p_{r}^{*}, 1\right)$ and satisfies the constraint $\gamma_{r a}=\gamma_{s r}$.

In situations here both non-forwarding and forwarding equilibriums exist, the following algorithm converges to the forwarding equilibrium:

The Strategic Game Algorithm:

1. Maximize $U_{s}\left(p_{s}, l\right)$ without constraints

2. Maximize $U_{r}\left(p_{r}, k\right)$ as in (14)

3. While (true)

4. Maximize $U_{s}\left(p_{s}, l\right)$ as in (14)

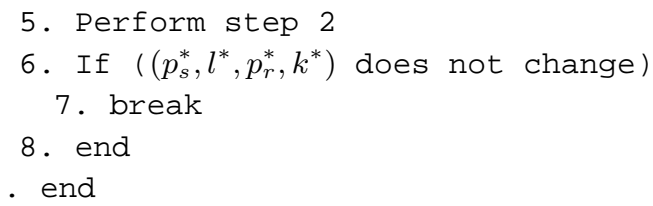

This algorithm converges in exactly two iterations. This is due to the restriction of discrete choices on forwarding/protocol preferences. To summarize, in this section we analyzed the strategic game component of our pricing mechanism. The strategic game is the major component of the pricing mechanism, depicted in Fig. 1.

\section{Convergence of the Pricing Mechanism}

Recall that our pricing mechanism consists of not only the source-relay strategic game, but also the utility maximizing source. Given the price per unit of service, the entire process converges at the reimbursement price that maximizes the utility of the source. We propose an algorithm that iteratively determines this price. Initially, the source sets $\mu=0$ and the users engage in a strategic game. The algorithm from the previous section determines the users' best responses for this game. The source finds its resulting utility and then sets $\mu=\mu+\Delta \mu$. The process repeats until either the source reaches its maximum value of $\mu$ (i.e., the algorithm converges to the non-forwarding case) or to a source utility value that is strictly less than the value of the previous iteration (i.e., the algorithm converges to the cooperative case).

\section{NumericAl Results}

\section{A. Example 1: Two User and Single AP Network}

Consider a network in which the AP is fixed at the origin and the potential relay is fixed 4 meters north of the AP. We compare the multi-hop and AF forwarding behavior of our pricing algorithm for different locations of the source. As in [11], the pricing game induces cooperation depending on the relative locations of the source and relay. The parameters for this simulation are borrowed from [11]: number of bits per frame $M=80$, bandwidth $W=10^{6} \mathrm{~Hz}$, noise variance $N o W=5 \times 10^{-15}$ Watts, $\operatorname{BER}(\gamma)=1 / 2 \exp (-\gamma / 2)$ for noncoherent frequency shift keyed (FSK), and a path gain formula given by $h=1 / d^{2}$, where $d$ is the distance between the transmitter and receiver in meters.

Figures 2 and 3 show the forwarding regions induced by pricing for multi-hop and AF transmission respectively. The not applicable (NA) region in both figures is a result of the initial routing assignments in the network - the relay node is always the node closer to the AP. The source node, therefore, is never located inside the NA region. We observe from Figs. 2 and 3 that the pricing algorithm stimulates cooperation at intuitively reasonable network geometries (i.e., geometries where the users are located relatively close to each other). The explanation for this is that if the inter-user path gain, $h_{s r}$, is large enough relative to $h_{s a}$, the source has a strong willingness to pay the relay to forward its data. As a result, the relay has a stronger tendency to forward data. The forwarding region is slightly larger for AF transmission, 


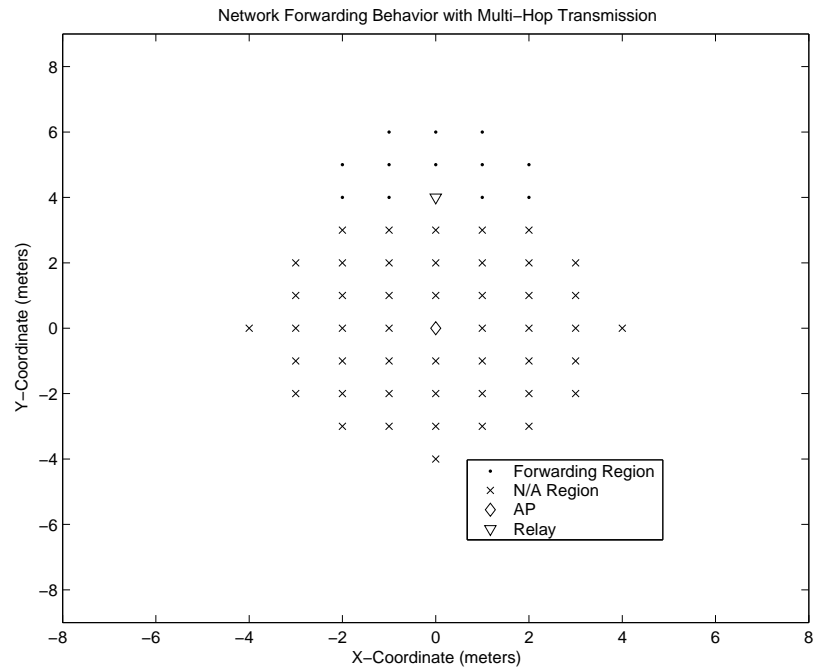

Fig. 2. Network Forwarding Behavior with multi-hop transmission

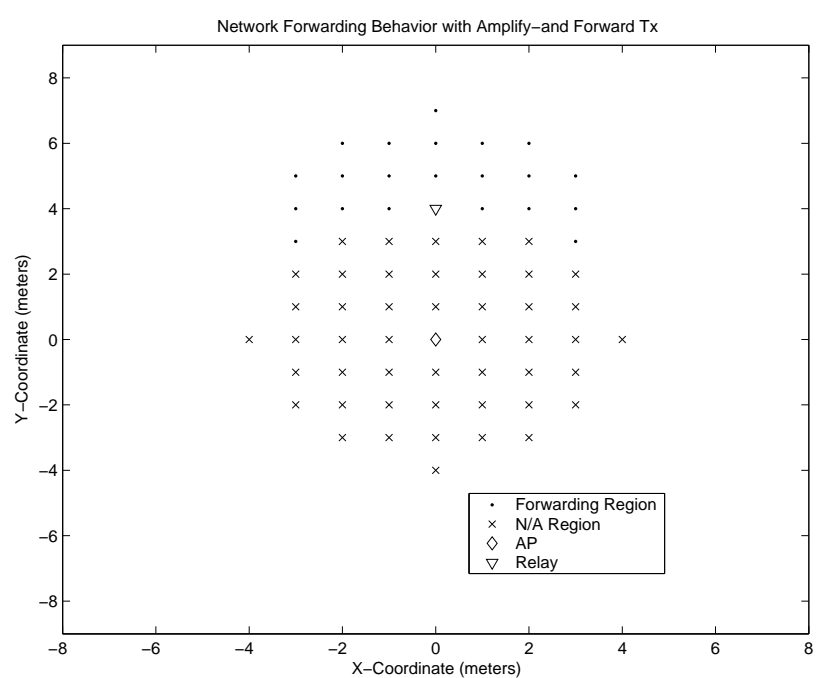

Fig. 3. Network Forwarding Behavior with AF cooperation

despite reducing its performance through the received SNR approximation $\gamma_{A F}=\gamma_{s a}+\min \left\{\gamma_{s r}, \gamma_{r a}\right\}$. This is expected since $\mathrm{AF}$ transmission is by far the more energy efficient alternative. Users receive a higher utility using AF since they can transmit a larger amount of data for a given energy cost.

We next compare the AP revenue, source utility and relay utility of our pricing system to a non-forwarding system. In the non-forwarding system, $\mu$ is identically set to zero (i.e., there exists no forwarding incentives for the relay). As a result, the relay and the source choose $k=0$ and $l=0$ respectively. The Nash equilibrium of this strategic game has the action profile $\left(p_{s}^{*}, 0, p_{r}^{*}, 0\right)$. The AP chooses the value of $\lambda$ such that its revenue, $\lambda W\left[f\left(\gamma_{s a}^{*}\right)+f\left(\gamma_{r a}\right)^{*}\right]$, is maximized. Consider the vertical line passing through the origin in Fig. 2. Figures [4-6] plot the revenue, source utility and relay utility respectively of both systems along this line, north of the relay. The revenue, $U_{s}$ and $U_{r}$ are higher in the pricing system than

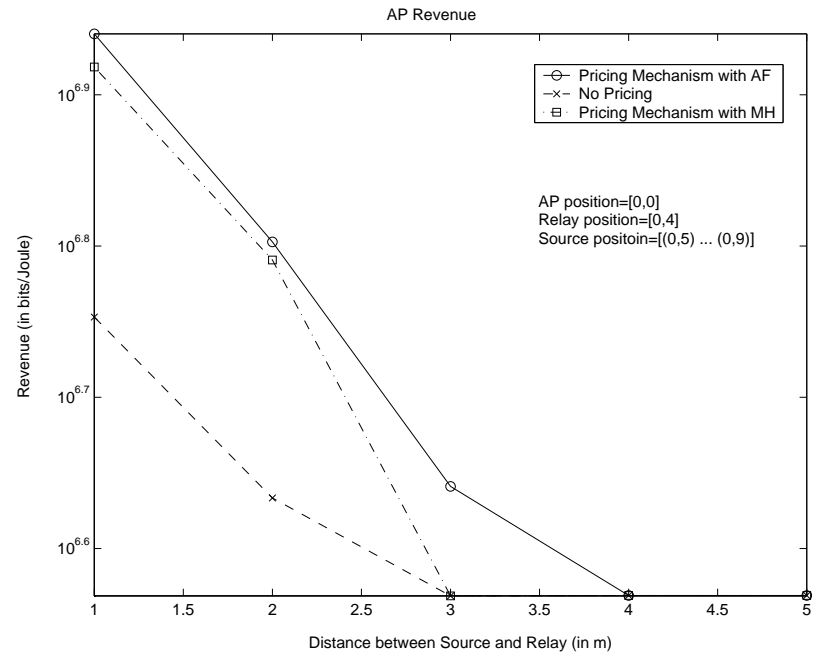

Fig. 4. AP Revenue in a two user network

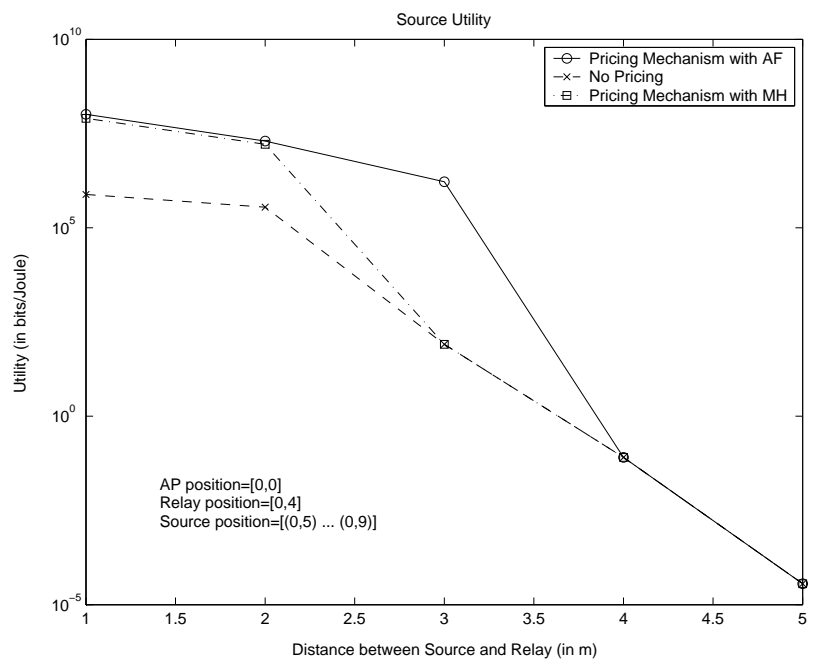

Fig. 5. Source Utility in a two user network

in the non-forwarding system. Thus it is clear all all parties (i.e., AP, source and relay) benefit from the use of the pricing mechanism.

From Fig. 5, in the context of our pricing algorithm, we see the source's utility is only slightly improved when using the AF protocol compared to multi-hop transmission. This is because when using the AF protocol the received SNR is approximated to equal $\gamma_{A F}=\gamma_{s a}+\min \left\{\gamma_{s r}, \gamma_{r a}\right\}$. From this expression, when the source is close to the relay relative to the AP, the term $\gamma_{s r}$ dominates $\gamma_{s a}$. Consequently, the source receives similar levels of satisfaction in using both the $\mathrm{AF}$ and multi-hop protocols. However, there is a gain in the overall system performance (not evaluated here) because AF provides significantly greater energy efficiency.

\section{B. Example 2: Single AP with Multiple Users}

Consider a network in which users are randomly and uniformly distributed in a $10 \mathrm{~m} \times 10 \mathrm{~m}$ region with the AP 


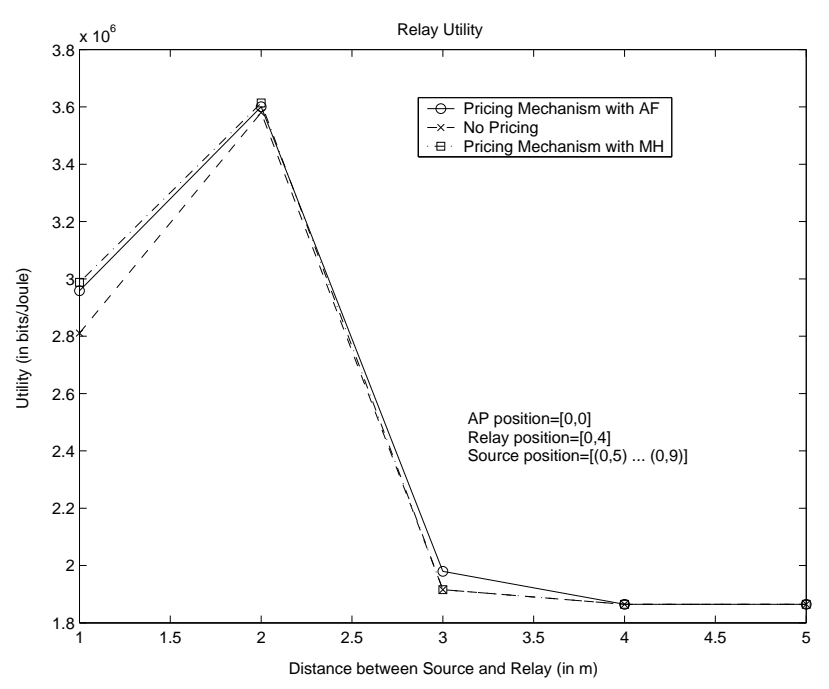

Fig. 6. Relay Utility in a two user network

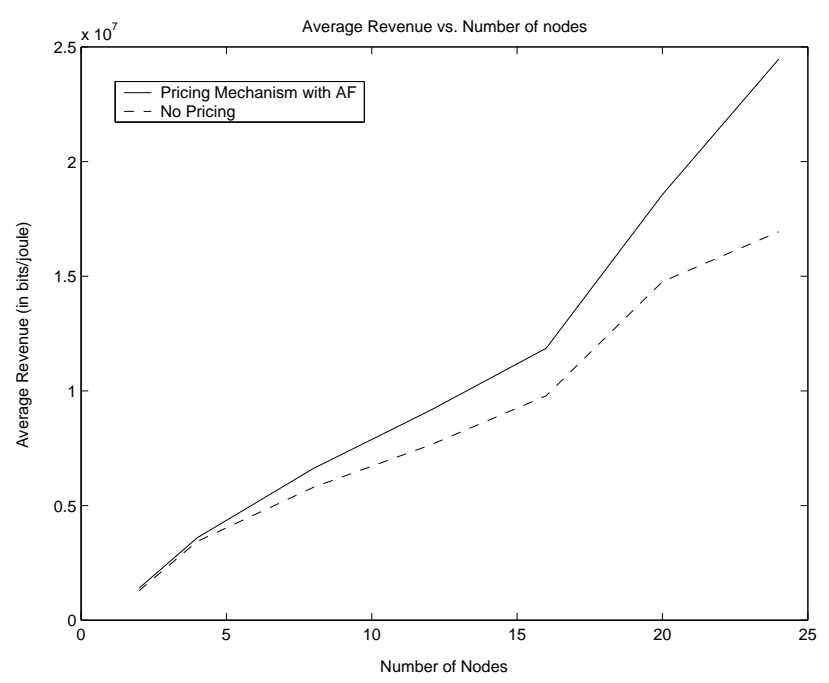

Fig. 7. Access Point's Revenue versus Number of Nodes in the Network

located at its center. We simulate the AP's average revenue as a function of the number of nodes in the network using our pricing mechanism and the non-forwarding system. All simulations average over 100 network realizations. The same parameters are used as in the previous subsection.

From Fig. 7, we observe that the impact of our pricing scheme on the AP's revenue increases with the number of nodes in the network. Networks consisting of a few randomly distributed nodes do not benefit much from our pricing mechanism. This is because our mechanism requires nodes to be relatively close together in order to provide beneficial results. Thus, on average, our mechanism in these networks converges to the results of the non-forwarding system. However, with increasing density, the average distance between nodes decreases. Cooperative behavior is then induced more often, resulting in increased revenue generated by the AP.

\section{Conclusions}

This paper has presented a pricing mechanism that induces cooperation in commercial wireless ad hoc networks. We have considered cooperation by means of multi-hop transmission and cooperative diversity. We have shown that all parties benefit from our pricing mechanism when compared to a nonforwarding system. To our knowledge, we are the first to consider cooperative diversity in such a framework.

The analysis here showed that cooperative Nash equilibria only exist at network geometries where users are located relatively close to each other. Consequently, the impact of our pricing scheme is beneficial in networks of high densities, or in sparse networks where users are clustered in local groups. Also, despite degrading the performance of the AF protocol via the received SNR approximation, we have shown that it still outperforms both direct and multi-hop transmission.

\section{REFERENCES}

[1] A. Sendonaris, E. Erkip, and B. Aazhang, "User cooperation viversity parts I,II,", IEEE Transactions on Communication, vol. 51, pp. 1927 48, November 2003.

[2] J. Laneman, G. Wornell, and D. N. C. Tse, "Energy-Efficient Antenna Sharing and Relaying for Wireless Networks," Proc. IEEE WCNC, pp. 7-12, March 2000.

[3] M. Felegyhazi, J. P. Hubaux, and L. Buttyan, "Nash Equilibria of Packet Forwarding Strategies in Wireless Ad Hoc Networks," to appear in IEEE Transactions on Mobile Computing, 2005.

[4] S. Marti, T. J. Giuli, K. Lai, and M. Baker, "Mitigating Routing Misbehavior in Mobile Ad Hoc Networks," in Proc. The Sixth International Conference on Mobile Computing and Networking, pp. 255 - 265, August 2000.

[5] S. Buchegger and J. Y. LeBoudec, "Performance Analysis of the CONFIDANT Protocol: Cooperation Of Nodes - Fairness in Distributes Ad-hoc Networks," in Proc. of the IEEE/ACM Workshop Mobile Ad Hoc Networks, pp. 226 - 236, June 2003.

[6] P. Michiardi and R. Molva, "CORE: A Collaborative Reputation Mechanism to Enforce Node Cooperation in Mobile Ad Hoc Networks," 6th IFIP Conference on Security, Communications and Multimedia, September 2002.

[7] L. Buttyan and J. P. Hubaux, "Stimulating Cooperation in self-organizing mobile ad hoc networks," ACM/Kluwer MONET, vol. 8, pp. 579 - 592, October 2003

[8] S. Zhong, J. Chen, and Y. R. Yang, "Sprite: A Simple, Cheat-Proof, Credit-Based System for Mobile Ad-Hoc Networks," In Proc. of IEEE INFOCOM, 2003.

[9] J. Crowcroft, R. Gibbens, F. Kelly, and S. Ostring, "Modelling incentives for collaboration in mobile ad hoc networks," to appear in IEEE/ACM Transactions on Networking, vol. 57, pp. 427 - 439, August 2004.

[10] P. Marbach and Y. Qiu, "Cooperation in Wireless Ad Hoc Networks: A Market-Based Approach," to appear in IEEE/ACM Transactions on Networking, 2005.

[11] O. Ileri, S. C. Mau, and N. B. Mandayam, "Pricing for Enabling Forwarding in Self-Configuring Ad Hoc Networks," IEEE Journal on Selected Areas in Communications, vol. 23, pp. 151- 162, January 2005.

[12] M. J. Osborne, An Introduction to Game Theory. New York, NY: Oxford University Press, 2004.

[13] C. U. Saraydar, N. B. Mandayam, and D. J. Goodman, "Efficient Power Control via Pricing in Wireless Data Networks," IEEE Transactions on Communications, vol. 50, pp. 291-303, February 2002. 